\title{
Belgeo
}

Revue belge de géographie

\section{Entre naturalisation de la montagne et patrimonialisation identitaire : l'ambivalence des représentations du Cantal dans les topoguides de randonnée}

Between mountain naturalization and identity patrimonialization: ambivalence

of the representations of the Cantal in topographical hiking guides

\section{Mauricette Fournier}

\section{(2) OpenEdition}

1 Journals

\section{Édition électronique}

URL : http://journals.openedition.org/belgeo/7178

DOI : $10.4000 /$ belgeo. 7178

ISSN : 2294-9135

Éditeur :

National Committee of Geography of Belgium, Société Royale Belge de Géographie

\section{Référence électronique}

Mauricette Fournier, «Entre naturalisation de la montagne et patrimonialisation identitaire :

l'ambivalence des représentations du Cantal dans les topoguides de randonnée», Belgeo [En ligne],

3 | 2012, mis en ligne le 18 mars 2013, consulté le 19 avril 2019. URL : http://journals.openedition.org/ belgeo/7178; DOl : 10.4000/belgeo.7178

Ce document a été généré automatiquement le 19 avril 2019.

Belgeo est mis à disposition selon les termes de la licence Creative Commons Attribution 4.0 International. 


\section{Entre naturalisation de la montagne et patrimonialisation identitaire : l'ambivalence des représentations du Cantal dans les topoguides de randonnée}

Between mountain naturalization and identity patrimonialization : ambivalence

of the representations of the Cantal in topographical hiking guides

Mauricette Fournier

\section{Introduction}

1 Cet article se propose d'examiner le rôle des topoguides de randonnée dans la construction de l'image du Cantal, département de moyenne montagne faiblement peuplé situé au cœur du Massif central. Il se situe à l'articulation de plusieurs questionnements : les modalités de développement du tourisme diffus, les processus de patrimonialisation, le rôle des guides et notamment de leur iconographie dans la fabrication de l'imaginaire des lieux (Bédard, Augustin et Desnoilles, 2012).

2 Le tourisme diffus (nommé également tourisme "vert", tourisme "doux") "rassemble différentes formes non concentrées de tourisme qui existent dans des espaces non touristiques, au sein d'économies polyvalentes dont la dynamique peut être déclinante ou ascendante" (Knafou, 1995). De fait, c'est une forme de tourisme que les instances politiques, du niveau local à l'échelle européenne, cherchent à promouvoir dans les milieux ruraux afin de prendre le relais d'une activité agricole de plus en plus malthusienne. C'est un "tourisme d'espaces" (Vitte, 2001) qui, ne nécessitant pas d'investissements lourds, cherche à assurer son développement en valorisant un patrimoine de "supports" naturels propice à la pratique des loisirs sportifs (Bourdeau, 1995 ; Corneloup et Perrin-Malterre, 2009), parmi lesquels 
la randonnée est devenue au cours des dernières décennies l'une des activités les plus pratiquées.

3 Anciennes voies de communication et d'échanges longtemps associées aux activités agricoles, les chemins, qui ont constitué un élément structurant des paysages agraires, semblaient condamnés dans les années soixante-dix, en raison des mutations de l'activité agricole. Au cours de la décennie suivante, prenant conscience qu'aliénations et suppressions mettaient en danger ces biens collectifs alors même qu'émergeait une demande croissante de tourisme vert, les législateurs français ont proposé un outil réglementaire : promu en 1983, le Plan Départemental des Itinéraires de Promenade et de Randonnée (PDIPR) eut pour objectif d'assurer la protection d'une partie des chemins ruraux grâce à la création et au développement des itinéraires de randonnée (Greveche, 2002). Dès lors, contraints d'élaborer ces plans, les départements ruraux ont d'autant plus volontiers contribué à l'aménagement des sentiers et à l'essor de la randonnée qu'ils voyaient dans cette nouvelle manière de pratiquer la marche un levier de développement pour leurs territoires.

4 Comme le souligne O. Etcheverria (1999), "le déploiement géographique des sentiers de randonnée illustre donc le passage d'une logique de production et de circulation à une logique de consommation" qui se nourrit de la diversité régionale au travers des paysages et des patrimoines. C'est pourquoi la pratique - croissante - de la randonnée a contribué à "l'explosion patrimonialisante" (Gravari-Barbas et Guichard-Anguis, 2003; Heinich, 2009), qu'il s'agisse de patrimonialiser la nature (Duval, 2007 ; Guichard-Anguis et Héritier, 2008; Dérioz, 2010; Gauchon, 2010, Sénil, 2011) ou encore de redécouvrir le petit patrimoine rural, promu au rang de ressource à part entière pour le développement des territoires (Landel, 2007). Les topoguides vont alors constituer un rouage essentiel du processus de patrimonialisation. Si leur principale fonction consiste à décrire les itinéraires, ils vont rapidement s'enrichir de nombreuses photographies et informations sur les lieux parcourus, participant d'une part à la construction des identités territoriales, d'autre part à celle des imaginaires géographiques destinés à un touriste qui "demande plus qu'avant des mises en scènes lui donnant envie de vivre des expériences culturelles par la rencontre intense de la nature, des autres et des locaux [...] à la recherche de cette ruralité conviviale et humaine" (Corneloup et Perrin-Malterre, 2009).

L'article se propose d'illustrer cette ambivalence en s'appuyant sur les divers topoguides de randonnée du département du Cantal ; tout d'abord en étudiant l'offre d'itinéraires et sa répartition géographique; puis en faisant le décompte des thématiques abordées dans les ouvrages et l'analyse des documents iconographiques afin d'envisager ce qui relève de la patrimonialisation identitaire et des représentations (notamment de la nature montagnarde) plus spécialement destinées à la clientèle touristique. C'est aussi l'occasion de présenter Chamina qui fut, dans le Massif Central, l'un des acteurs majeurs du développement de la randonnée, du tourisme vert et de la redécouverte du patrimoine vernaculaire. 


\section{Une offre abondante d'itinéraires privilégiant les hautes terres}

\section{L'importance de l'offre}

Il existe dans le Cantal deux principaux types de sentiers pour la promenade pédestre : les chemins de Petite Randonnée (PR) et les sentiers de Grande Randonnée (GR). Les plus anciens, les GR, dont une variante est constituée par les GR de Pays, sont conçus et gérés par la Fédération Française de Randonnée Pédestre (FFRP) et correspondent tous deux à une marque déposée. Reconnaissable à son balisage rouge et blanc, le GR permet de traverser une région en plusieurs jours. Le GR de Pays, balisé en rouge et jaune, a pour objectif de favoriser la découverte d'un territoire homogène (parc naturel, vallée..). Trois GR, cumulant un total de $602 \mathrm{~km}$, permettent de découvrir le Cantal : le GR 4, qui effectue une grande traversée du Massif Central ; le GR 400, concentré sur les Monts du Cantal et leurs principales vallées (Alagnon, Cère, Jordanne, Rhue, Mars) et le GR Pays "Volcan du Cantal, Pays de Saint- Flour et de Ruyne-en-Margeride" (tableau 1 et figure 1).

Tableau 1. Les sentiers de Grande Randonnée de la FFRP dans le Cantal.

\begin{tabular}{|c|c|c|c|}
\hline & Types de sentiers & $\begin{array}{l}\text { Nombre de } \\
\text { kilomètres }\end{array}$ & Détail des boucles \\
\hline GR4 & linéaire & 140 & \\
\hline GR 400 & 5 boucles & 168 & $\begin{array}{c}42 ; 35,7 ; 33 ; 35,5 \\
22 \mathrm{~km}\end{array}$ \\
\hline GR Pays & 3 boucles & 294 & $105 ; 102 ; 87 \mathrm{~km}$ \\
\hline Total & & 602 & \\
\hline
\end{tabular}

Figure 1. Les sentiers de Grande Randonnée de la FFRP dans le Cantal.

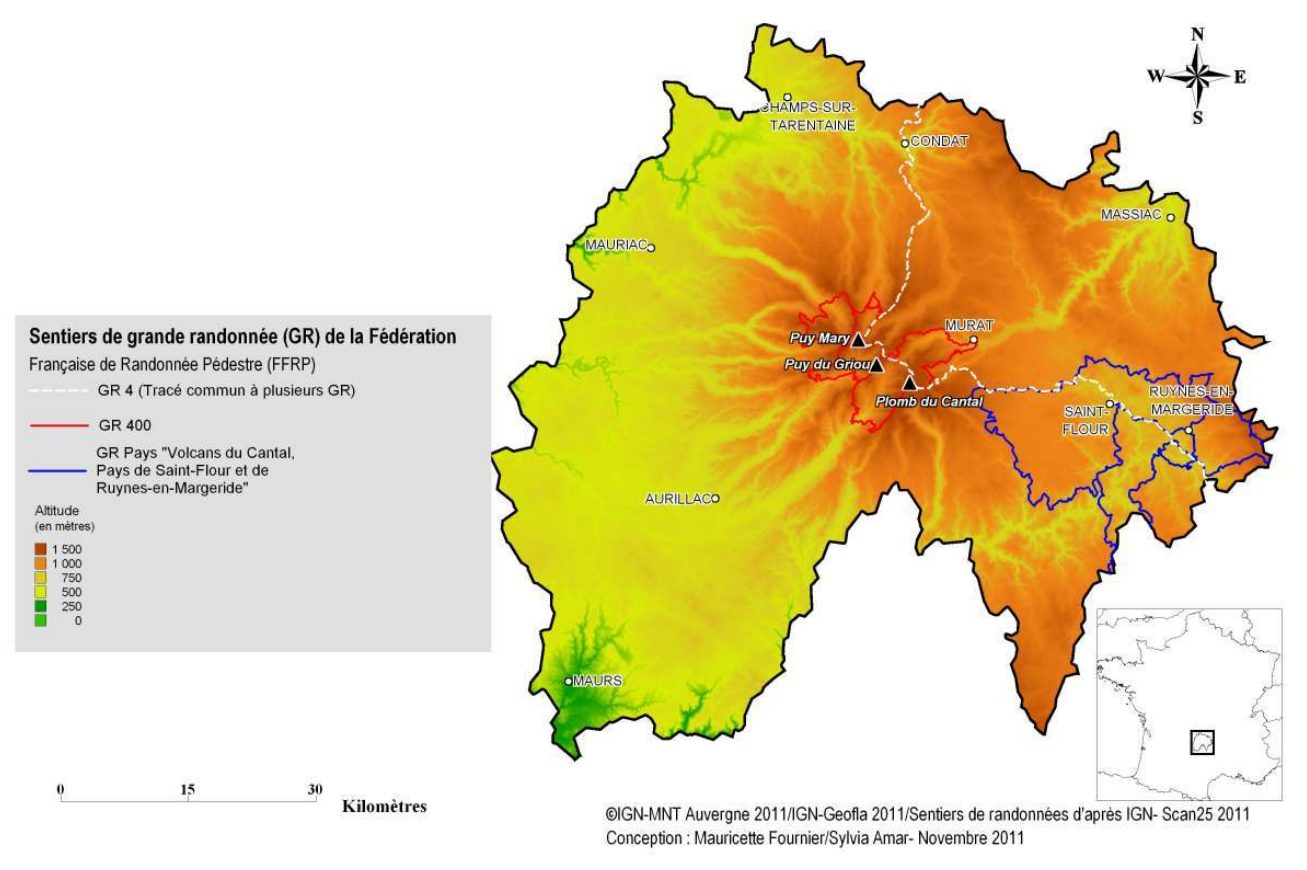


7 Apparus plus récemment, les chemins de Petite Randonnée constituent toujours une boucle autour d'un point de départ ; ils sont balisés de différentes couleurs (en général bleu, jaune, vert) selon la durée du parcours. Le recensement des PR à l'échelle d'un territoire est très difficile car ils relèvent d'initiatives nombreuses, variées, rarement coordonnées. Seuls ont été retenus ici les itinéraires repris dans les topoguides des éditions Chamina et ceux de la collection "Promenades et Randonnées au Pays des Grands Espaces" publiés par le Conseil Général du Cantal dans le cadre de sa politique de promotion de la randonnée pédestre. A donc été exclue la multitude de brochures, cartes, dépliants créés notamment par des offices de tourisme ou des associations locales. Concernant tout ou partie du département, on ne dénombre pas moins de cinq ouvrages aux éditions Chamina : "Le Cantal" et "Le Volcan Cantalien", totalement circonscrits dans les limites départementales, auxquels s'ajoutent "Margeride et Gévaudan", "Aubrac" et "Monts du Cézallier", qui proposent également des randonnées dans les départements limitrophes ${ }^{1}$ . La collection "Promenades et Randonnées au Pays des Grands Espaces" a pour objectif de regrouper toutes les PR inscrites au Plan Départemental des Itinéraires de Promenade et Randonnée (PDIPR); elle se compose de dix topoguides ${ }^{2}$, représentant au total 248 itinéraires (2224,8 km cumulés).

Tableau 2. Topoguides de Petites Randonnées dans le département du Cantal.

\begin{tabular}{|c|c|c|c|c|c|}
\hline Liste des topoguides & \multicolumn{4}{|c|}{ Nombre de sentiers } & \multirow{2}{*}{$\begin{array}{c}\text { Nombre de } \\
\mathrm{km}\end{array}$} \\
\hline Collection CHAMINA & bleu & jaune & vert & Total & \\
\hline Le Cantal & 6 & 16 & 18 & 40 & 422,3 \\
\hline Le Volcan Cantalien & 3 & 24 & 22 & 49 & 539,5 \\
\hline Monts du Cézallier & 10 & 11 & 5 & 26 & 225 \\
\hline Aubrac & 2 & 3 & 2 & 7 & 59,5 \\
\hline Margeride et Gévaudan & 6 & 9 & 2 & 17 & 132 \\
\hline Total & 27 & 63 & 49 & 139 & 1378,3 \\
\hline \multicolumn{6}{|l|}{$\begin{array}{l}\text { Collection "Promenades et } \\
\text { Randonnées au Pays des Grands } \\
\text { Espaces" }\end{array}$} \\
\hline Pays de Saint-Flour & 1 & 14 & 9 & 24 & 264 \\
\hline $\begin{array}{l}\text { Pays de la Pinatelle et de la Haute } \\
\text { Santoire }\end{array}$ & 4 & 5 & 4 & 13 & 138 \\
\hline Pays de Massiac & 7 & 17 & 9 & 33 & 300 \\
\hline Pays de la Châtaigneraie & 24 & 24 & 9 & 57 & 510,8 \\
\hline $\begin{array}{l}\text { Le Pays entre Dordogne et Monts du } \\
\text { Cantal }\end{array}$ & 20 & 19 & 9 & 48 & 404 \\
\hline Plomb du Cantal et ses vallées & 7 & 12 & 9 & 28 & 226,8 \\
\hline Pays d'Aurillac & 5 & 8 & 1 & 14 & 114 \\
\hline Entre Plomb du Cantal et Aubrac & 3 & 6 & 4 & 13 & 127,7 \\
\hline $\begin{array}{l}\text { Les chemins des écoliers Truyère } \\
\text { Margeride }\end{array}$ & 3 & 9 & & 12 & 78,5 \\
\hline Volcans et vallées en Carladès & & 4 & 2 & 6 & 61 \\
\hline Total & 74 & 118 & 56 & 248 & 2224,8 \\
\hline TOTAL GENERAL & 101 & 181 & 105 & 387 & 3603,1 \\
\hline
\end{tabular}

Collections Chamina et "Promenades et Randonnées au Pays des Grands Espaces".

8 L'offre de randonnées pédestres du département du Cantal est donc très importante et diversifiée ; on dénombre $600 \mathrm{~km}$ de GR ou GR Pays et $3603 \mathrm{~km}$ de PR en additionnant les circuits de Chamina à ceux des éditions départementales (tableau 2). Il existe cependant quelques redondances qu'il faut soustraire (47 itinéraires identiques, soit $484 \mathrm{~km}$ ), pour arriver à un total de 330 PR totalisant 3119,1 km. Mais si l'offre paraît importante, elle n'est cependant pas répartie de façon homogène sur l'ensemble du territoire. 


\section{Une répartition inégale : une nette inclinaison pour la montagne pastorale}

9 Un examen rapide de la répartition par commune des itinéraires, tous topoguides confondus, montre qu'aucune partie du Cantal n'est véritablement dépourvue de cette offre de loisirs : en effet, 197 communes sur les 260 que compte le département sont parcourues par au moins un itinéraire de randonnée qu'il s'agisse de PR ou de GR (figure 2). Cependant, la cartographie des sentiers de randonnée révèle de nettes disparités spatiales. Tandis que la bordure occidentale (l'Artense, la Xaintrie, le nord-ouest de la Châtaigneraie) manifeste un certain sous-équipement, deux secteurs semblent concentrer les chemins de randonnée: le volcan cantalien et le pays de Massiac. On peut donc s'interroger sur ces disparités en examinant d'une part la typologie des sentiers de randonnée (PR ou GR) et d'autre part le statut (public ou privé) des éditeurs de topoguides.

La première constatation est que les GR sont majoritairement localisés au centre et à l'est du département. Ceci s'explique en partie par l'existence déjà ancienne du GR 4 qui relie la Méditerranée à l'Atlantique par le Massif Central : après avoir traversé la Margeride à l'est, le chemin de randonnée se poursuit sur la planèze de Saint-Flour, gravit les monts du Cantal avant de filer plein nord vers le Sancy. C'est bien l'environnement montagnard qui a été privilégié. Cet itinéraire a ensuite servi de support à la FFRP pour créer de nouveaux sentiers de grande randonnée: les boucles du GR 400 (Hauts plateaux et Monts d'Auvergne) et du GRP (Volcan du Cantal, Pays de Saint-Flour et de Ruynes-en-Margeride) présentent ainsi d'importants tronçons communs avec le GR 4.

11 Pour ce qui est des PR, l'éditeur Chamina marque également une nette préférence pour les massifs montagneux du département - Monts du Cantal, Aubrac, Cézallier, Margeride - ce qui le conduit à privilégier l'est et le centre plutôt que l'ouest aux altitudes plus modérées. La maison d'édition montre par ces choix qu'elle cherche à répondre aux attentes de sa clientèle. Diverses enquêtes réalisées auprès des randonneurs à l'échelle nationale ou départementale (Foussat, 2004) indiquent en effet qu'une majorité d'entre eux préfère pratiquer cette activité en montagne plutôt qu'à la campagne ou en plaine. Le massif du Cantal, avec des dénivelés relativement modérés et des temps de parcours raisonnables pour atteindre les crêtes, présente de nombreux atouts pour le développement de cette activité sportive de pleine nature, qui offre à ceux qui la pratiquent une "expérience paysagère non linéaire" (Niel et Sirost, 2008). 
Figure 2. Les sentiers de petite randonnée des topoguides Chamina (répartition par communes).

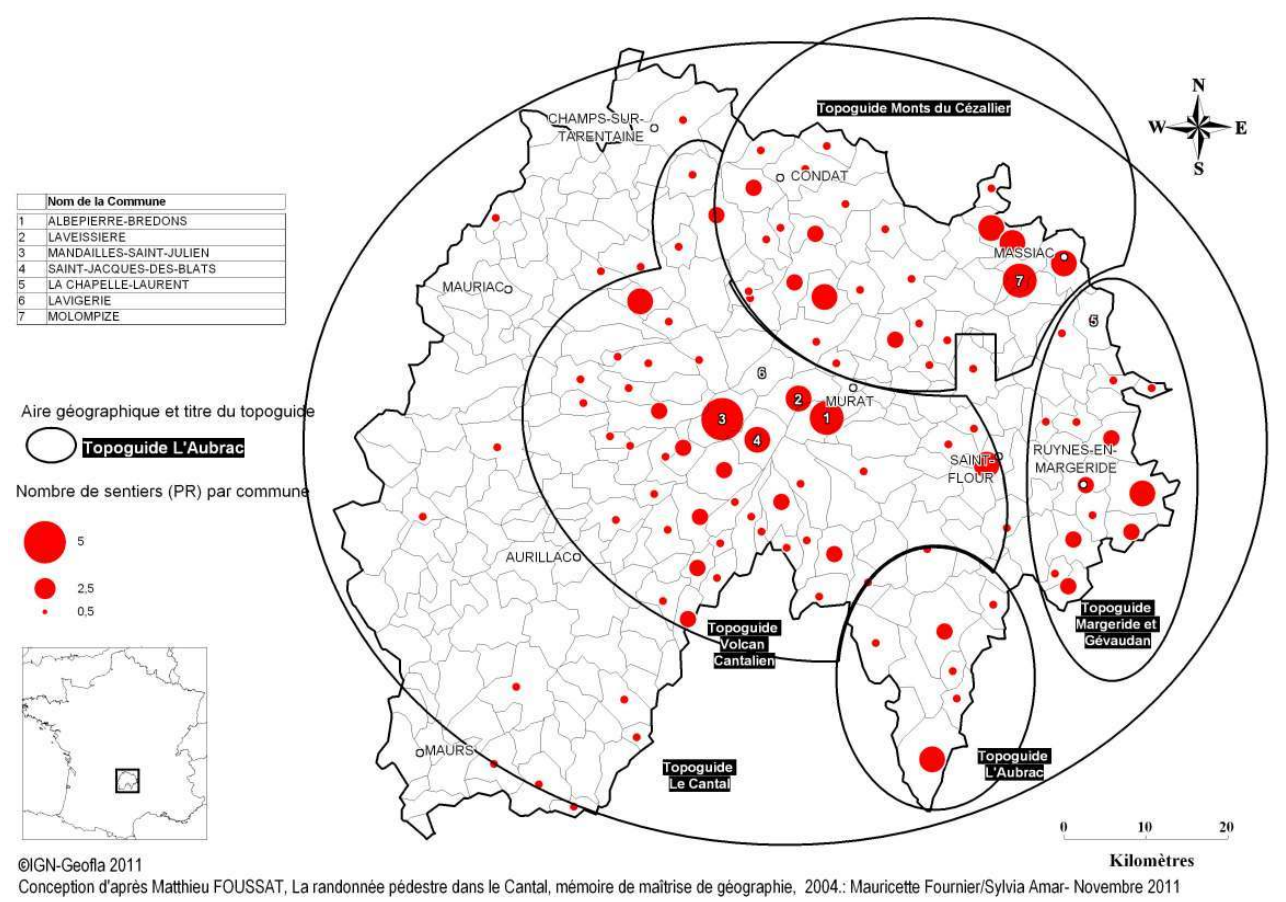

Les topoguides de la collection "Promenades et randonnées au pays des grands espaces", conçus dans le cadre du PDIPR du Cantal, dont l'ambition est d'atténuer les disparités entre les différentes régions du département, sont répartis de manière plus homogène. Les espaces occidentaux du Cantal - la Châtaigneraie, le Mauriacois - bénéficient d'une couverture éditoriale, même si l'on relève encore une certaine surreprésentation du Plomb du Cantal et de ses vallées.

C'est cependant sans surprise que l'on relèvera au final, pour l'ensemble des topoguides, la surreprésentation des dix-huit communes du volcan cantalien; elles concentrent 63 itinéraires de petite randonnée (soit près de $19 \%$ du total des sentiers du Cantal). Entre elles apparaît également un gradient, selon leur proximité avec le cœur du massif ; ainsi Laveissière (siège de la station de ski du Lioran, point d'attraction hivernal comme estival grâce à la présence de remontées mécaniques fonctionnant aussi l'été) compte quatorze itinéraires ; il y en a huit à Albepierre-Bredons et six à Saint-Jacques-des-Blats, communes situées au pied du Plomb du Cantal, point culminant du massif (1 $855 \mathrm{~m}$ ); on en dénombre encore huit à Mandailles-Saint-Julien et six à Lavigerie, localisées de part et d'autre du Puy Mary, Grand Site National qui attire annuellement plus de 500000 visiteurs.

\section{L'ambivalence des représentations}

\section{La valorisation des patrimoines identitaires grâce à une approche originale de Chamina}

Les topoguides ne se contentent pas de proposer des parcours de randonnée, d'apporter des informations techniques; ils fournissent aussi à leurs utilisateurs une documentation parfois très riche pour appréhender les patrimoines locaux. C'est tout particulièrement le 
cas des éditions Chamina, engagées très précocement tant dans la création de sentiers de randonnée que dans celle des topoguides qui les accompagnent.

Créée en 1974, à l'initiative de Bernard Quinsat, l'association Chamina a été un acteur majeur du développement de la randonnée, du tourisme diffus et de la redécouverte du petit patrimoine dans le Massif Central. Ses premières actions ont consisté à aménager des gîtes d'étape le long des chemins de grande randonnée. Souhaitant démocratiser et rendre plus accessible la pratique de cette activité, ses membres ont inventé la notion de petite randonnée : des parcours en boucle à la journée, variant d'une à plusieurs heures. $\mathrm{Au}$ total, l'association aura créé, balisé, développé et aménagé plus de $15000 \mathrm{~km}$ d'itinéraires dans le Massif Central. Cependant, pour les responsables de Chamina le développement de ce loisir de plein air qu'est la randonnée, ne constituait pas le seul objectif. Il s'agissait surtout de valoriser les territoires ruraux traversés en favorisant la création d'activités économiques pérennes dans le domaine du tourisme. L'action de Chamina, soutenue par les élus locaux, s'inscrivait clairement dans une logique de création d'une offre touristique.

Ceci a conduit l'association à se diversifier dans le domaine du tourisme et de l'édition. En 1978, Chamina a créé une association de tourisme devenue plus tard une véritable agence de voyages, Chamina-Sylva, qui élabore et commercialise des produits à l'échelle mondiale. En 1976 est édité " 25 circuits dans les Dômes", premier topoguide d'une collection qui comptera 120 titres au moment de la faillite financière de l'entreprise en $2008^{3}$. Opérateur reconnu, régulièrement sollicité aussi bien pour son savoir-faire technique en matière de conception d'itinéraire et de balisage que pour l'édition de topoguides de qualité, la maison d'édition Chamina s'était professionnalisée ${ }^{4}$ sans perdre de vue sa philosophie initiale qui consistait à considérer la marche comme "un moyen de réapprendre et de mieux connaître les milieux que l'on traverse". 
Figure 3. Les topoguides Chamina, des encyclopédies du patrimoine local (modèles-type des plans de montage : pages thématiques et pages de présentation des PR).
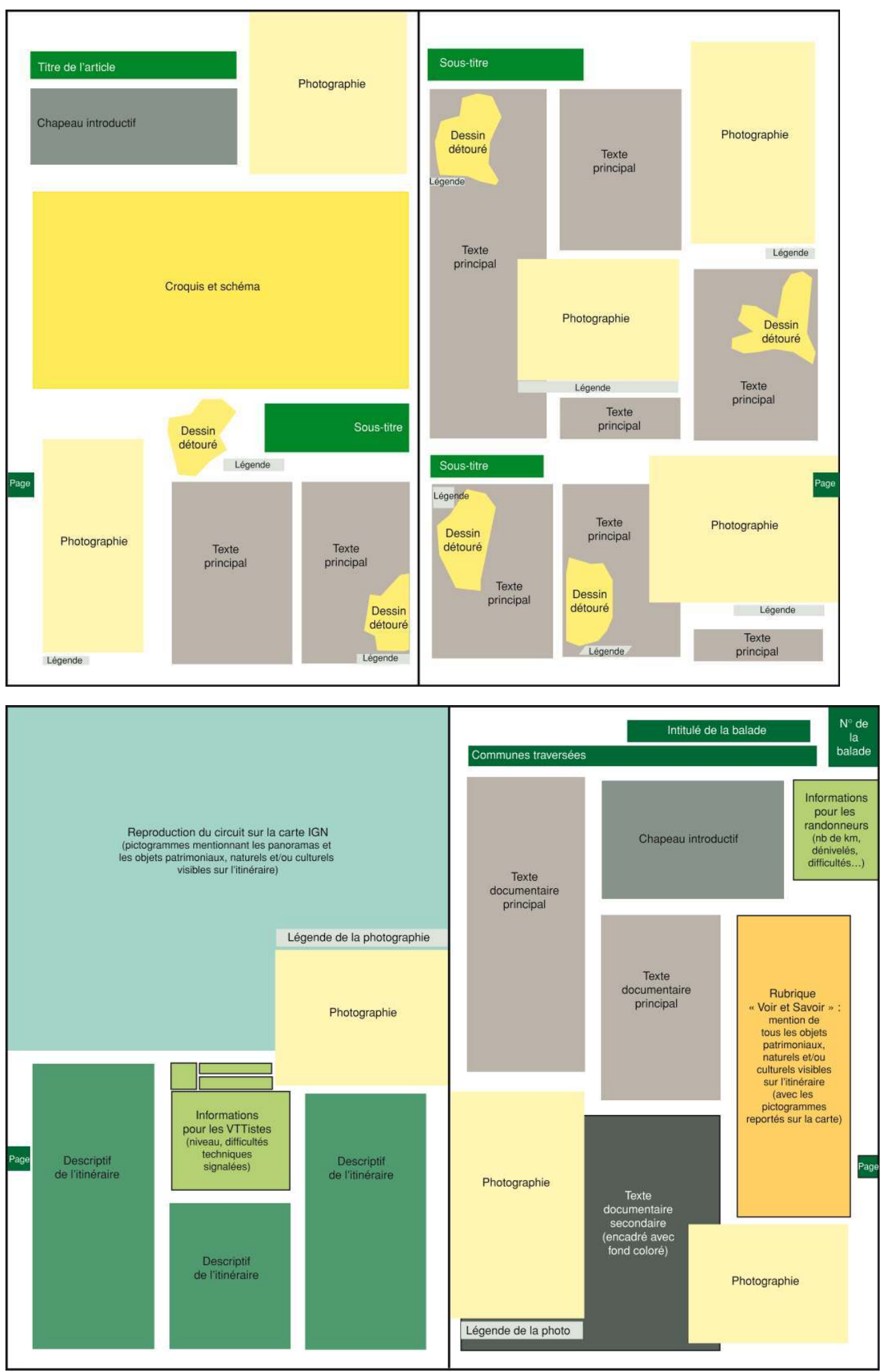

17 La maison d'édition a ainsi toujours manifesté un très grand souci pour mettre en valeur le patrimoine des communes rurales, et faire de ses topoguides de véritables encyclopédies à une échelle micro-locale. Pour parvenir à cet objectif, elle avait mis au point des pratiques éditoriales originales, mobilisant à la fois les habitants des territoires 
concernés pour le recensement des particularités locales et des spécialistes (scientifiques, universitaires) pour la rédaction des synthèses thématiques. Dès le démarrage d'un projet, une fiche très détaillée était adressée à toutes les mairies incluses dans le périmètre du topoguide à venir afin que soit répertorié tout ce qui pouvait présenter un intérêt patrimonial (d'ordre naturel ou culturel). Des réunions étaient ensuite organisées avec les représentants des collectivités et du milieu associatif dans le but de valider un synopsis général à partir des informations recueillies et de la documentation existante. Enfin, le secrétaire d'édition se mettait en quête des spécialistes pouvant rédiger les pages thématiques retenues à l'issue de ces réunions. La société locale, par l'intermédiaire des élus, des chargés du développement, des responsables associatifs, a donc eu la possibilité de participer à la sélection des objets mis en lumière dans le topoguide, les élevant au statut d'objets patrimonialisés, marqueurs de l'identité du territoire : elle a pris "ses lieux, ses objets, ses monuments comme des reflets intelligibles de son histoire, de sa culture" (Jeudy, 2008).

Cette richesse d'informations, les topoguides cherchent à en conserver l'exhaustivité en la distribuant dans les diverses rubriques de la maquette: "Voir et savoir", texte principal, chapeau, encarts, photographies, légendes développées de l'iconographie (figure 3). Le recensement de l'ensemble des points remarquables signalés dans la rubrique "Voir et savoir" de trois des topoguides Chamina permet de rendre compte de l'importance numérique de l'information documentée par les collectivités ou les associations locales : 200 références localisées avec un pictogramme sur la cartographie des circuits de PR pour "Le volcan cantalien", 120 pour "Les Monts du Cézallier", encore 69 pour "Le Cantal, coeur de massif" (tableau 3).

Tableau 3. Relevé détaillé des rubriques "Voir et savoir" de trois topoguides Chamina.

\begin{tabular}{|l|c|c|c|}
\hline & $\begin{array}{c}\text { Volcan } \\
\text { cantalien } \\
(\text { Chamina, } \\
2002)\end{array}$ & $\begin{array}{c}\text { Monts du } \\
\text { Cézallier } \\
\text { (Chamina, 2000) }\end{array}$ & $\begin{array}{c}\text { Le Cantal } \\
\text { (Chamina, 2010) }\end{array}$ \\
\hline Site ou curiosité & 19 & 27 & 12 \\
\hline Zone boisée & - & 4 & - \\
\hline Point de vue & 48 & 4 & 15 \\
\hline Mégalithe & 1 & 3 & - \\
\hline Ruines intéressantes & 8 & 8 & 2 \\
\hline Architecture religieuse & 33 & 25 & - \\
\hline Architecture militaire & 2 & 2 & 14 \\
\hline Architecture civile & 37 & 16 & 3 \\
\hline Bourg intéressant & 6 & 8 & - \\
\hline Château & 13 & 7 & 4 \\
\hline Site géologique & 11 & 9 & 4 \\
\hline Flore remarquable & 10 & 5 & 3 \\
\hline Hors circuit & - & - & 4 \\
\hline Autre & 12 & - & 3 \\
\hline Musée & - & 2 & $\mathbf{6 9}$ \\
\hline Total & $\mathbf{2 0 0}$ & $\mathbf{1 2 0}$ & - \\
\hline
\end{tabular}

19 Une fois le topoguide achevé, chaque mairie recevait copie des pages la concernant afin d'en valider le contenu (textes, photos..). Ainsi construits au plus près du terrain, tout en bénéficiant du regard et de la plume des spécialistes, conjuguant de ce fait étroitement connaissances distanciées du savant et pratiques vernaculaires des habitants, les topoguides Chamina sont devenus des ouvrages de référence indispensables pour la 
connaissance des lieux; ils ont ainsi contribué à l'invention du patrimoine des espaces ruraux de moyenne montagne (et notamment du petit patrimoine, lavoirs, fours banaux, métiers à ferrer, croix de chemins...). C'est pourquoi ils ont été largement achetés dans le Massif Central, y compris par des personnes ne pratiquant pas la randonnée, selon les témoignages du personnel de Chamina comme de celui de certains libraires des petites villes de la région.

La démarche très singulière de Chamina explique l'originalité des thématiques abordées (tableau 4). Quoiqu'importants, les textes centrés sur le patrimoine naturel (géologie, flore, faune..) sont proportionnellement nettement moins nombreux que dans les topoguides de la FFRP. A contrario dominent dans les éditions Chamina les thématiques qui relèvent de l'histoire locale. De même, sont légèrement surreprésentés par rapport aux éditions de la FFRP les textes concernant l'architecture vernaculaire et les traditions et savoir-faire (par exemple dans le topoguide "Le Cantal", la vie dans les burons et la fabrication de l'aligot, le travail des gabarriers, la fête de la châtaigne, le vignoble d'Entraygues et du Fel...) Encore n'a-t-on pas comptabilisé dans le tableau 4, les thématiques proposées dans les encarts ou les légendes développées des photographies, ce qui aurait conduit à accentuer le différentiel patrimoine naturel / patrimoine culturel.

Tableau 4. Les grandes thématiques de l'information documentaire proposée par les topoguides Chamina et FFRP *

\begin{tabular}{|c|c|c|c|}
\hline Thématiques & $\begin{array}{c}\text { Le Cantal } \\
\text { (Chamina, } \\
2001) \\
\text { en } \%\end{array}$ & $\begin{array}{c}\text { Volcan cantalien } \\
\text { (Chamina, 2002) } \\
\text { en \% }\end{array}$ & $\begin{array}{c}\text { Volcan du Cantal } \\
\text { (FFRP, 2007) } \\
\text { en \% }\end{array}$ \\
\hline Nature, environnement & 31,3 & 21,9 & 38,4 \\
\hline Histoire locale & 40,4 & 38,4 & 27,2 \\
\hline Architecture vernaculaire & 6,3 & 12,3 & 8,3 \\
\hline Traditions, savoir-faire & 9,8 & 12,3 & 5,3 \\
\hline $\begin{array}{c}\text { Economie (agriculture, } \\
\text { tourisme) }\end{array}$ & 12,2 & 15,1 & 20,8 \\
\hline TOTAL & $\mathbf{1 0 0 , 0}$ & $\mathbf{1 0 0 , 0}$ & $\mathbf{1 0 0 , 0}$ \\
\hline
\end{tabular}

* Ce classement ne prend en compte que les thématiques développées dans les pages de présentation générale et le texte d'accompagnement principal des pages présentant les itinéraires, à l'exclusion, le cas échéant, des encarts et légendes développées (voir figure 3).

Dans le Massif central, les topoguides édités par Chamina ont bien souvent été à l'origine de dynamiques de développement local s'appuyant conjointement sur l'aménagement de sentiers de randonnée et des dynamiques de patrimonialisation. Le Pays de Massiac, situé dans le nord-est du département du Cantal et traversé par l'autoroute A75, constitue un bon exemple pour illustrer ces démarches. Les collections de Chamina, tout comme celles du Conseil Général, sont venues renforcer la visibilité en matière de randonnées pédestres de ce secteur géographique. Centrée autour de la petite ville de Massiac, l'intercommunalité correspond au regroupement de 14 communes qui concentrent 42 chemins soit $12,5 \%$ des itinéraires pédestres du Cantal (pour 5,6\% des communes). Deux d'entre elles, Molompize et La Chapelle-Laurent, comptent chacune six chemins, ce qui les classe parmi les sept communes cantaliennes les plus richement dotées (figure 2). Ce n'est pas le caractère montagnard qui permet ici d'expliquer la surreprésentation des sentiers pédestres, mais plutôt le volontarisme politique. Dès la mise en place du PDIPR, les élus de la communauté de communes du Pays de Massiac ont conçu un réseau de chemins de randonnée cohérent afin de mettre en valeur le patrimoine bâti (religieux, 
médiéval et vernaculaire). Comme cela a été observé en d'autres lieux (Haschar-Noe, 2009), le développement de la randonnée et la patrimonialisation ont participé ici à l'énonciation d'un nouveau territoire politique; la dynamique intercommunale s'est alimentée de cette reconstruction de l'identité locale.

\section{Iconographie : le choix du patrimoine naturel et des paysages de montagne}

Dans les topoguides, les photographies sont omniprésentes, surtout dans les éditions Chamina (tant dans ses propres publications que dans celles du Conseil Général du Cantal dont la réalisation lui avait été en partie sous-traitée). Dans les doubles pages thématiques, il n'est pas rare de trouver six documents iconographiques (photographies, dessins, cartes), le record devant être détenu par celle intitulée "quelques objets de la foi" dans le topoguide "Le Cantal", illustrée de onze photographies et d'un dessin, principalement de modestes croix de chemin sculptées dans la pierre. Au total, on dénombre 199 illustrations dans "Le Cantal" et 149 dans "Le Volcan cantalien", édités par Chamina, pour 144 pages chacun, 112 photographies pour 92 pages dans "Le Plomb du Cantal et ses vallées", publié dans la collection départementale. Moins fourni, "Volcan du Cantal, Pays de Saint-Flour et de Ruynes-en-Margeride" de la FFRP en propose encore 59 pour 144 pages. Destinée à accompagner les textes, l'iconographie est le plus souvent en adéquation avec les thématiques développées. Aussi ne s'étonnera-t-on pas de l'importance des images se rapportant au patrimoine culturel dans les anciens guides Chamina, de l'ordre de $40 \%$, aussi bien en nombre de photographies qu'en surface cumulée des illustrations (exemple du "Volcan cantalien" dans le tableau 5).

Toutefois, le nombre de photographies est insuffisant pour mesurer la préférence accordée par le décideur (l'éditeur, la collectivité qui participe au financement de la publication) à un type de lieu plutôt qu'à un autre, de même que pour appréhender l'impact de ces images sur le lecteur. Pour cela, il faut aussi tenir compte des dimensions des illustrations : la photographie occupe-t-elle une pleine page ou se réduit-elle à une vignette? L'importance de l'iconographie en lien avec le patrimoine culturel apparaît alors toute relative. En effet, ces images, aussi belles soient-elles, sont plutôt de petit format, tandis que les paysages naturels occupent souvent une pleine page, voire une double page. Les images représentant le patrimoine culturel ont une fonction essentiellement documentaire tandis que le rôle attendu des photographies paysagères relève de la séduction. 
Tableau 5. Les thématiques de l'iconographie dans les topoguides Chamina en fonction de la superficie des illustrations $\left(\mathrm{en} \mathrm{cm}^{2}\right.$ ).

\begin{tabular}{|l|c|c|c|c|}
\hline & \multicolumn{2}{|l|}{$\begin{array}{l}\text { Le volcan cantalien } \\
\text { Chamina 2002) }\end{array}$} & \multicolumn{2}{c|}{$\begin{array}{l}\text { Cantal cœur de } \\
\text { massif } \\
\text { Chamina 2010) }\end{array}$} \\
\hline & $\begin{array}{l}\text { Superficie } \\
\text { En cm }\end{array}$ & $\%$ & $\begin{array}{c}\text { Superficie } \\
\text { En } \mathrm{cm}^{2}\end{array}$ & $\%$ \\
\hline PAYSAGES & & & & \\
\hline Paysages naturels & 1864,3 & 28,2 & 872,7 & 44,1 \\
\hline Dont montagne & 939,0 & 14,2 & 366,6 & 18,5 \\
\hline Dont eau, lacs, rivières & 361,5 & 5,5 & 188,8 & 9,5 \\
\hline Dont forêts & 126,0 & 1,9 & - & - \\
\hline Paysages bâtis & 812,4 & 12,4 & 311,4 & 15,7 \\
\hline PATRIMOINES & & & & \\
\hline Patrimoine naturel & 1467,0 & 22,2 & 414,7 & 21,0 \\
\hline Dont faune & 115,5 & 1,8 & 57,4 & 2,9 \\
\hline Dont flore & 370,5 & 5,6 & 13,2 & 0,7 \\
\hline Dont géologie & 119,0 & 1,8 & - & - \\
\hline Patrimoine culturel & 2455,9 & 37,2 & 378,8 & 19,2 \\
\hline Dont architecture religieuse & 421,6 & 6,4 & 55,3 & 2,8 \\
\hline Dont architecture vernaculaire & 932,0 & 14,2 & 203,9 & 10,3 \\
\hline Dont histoire locale & 199,0 & 3,0 & 69,9 & 3,5 \\
\hline Dont traditions, savoir-faire & - & - & 49,7 & 2,5 \\
\hline TOTAL & $\mathbf{6 5 9 9 , 6}$ & $\mathbf{1 0 0 , 0}$ & $\mathbf{1 9 7 7 , 6}$ & $\mathbf{1 0 0 , 0}$ \\
\hline
\end{tabular}

Une place particulière est alors accordée aux paysages de montagne. Prenons à nouveau comme exemple "Le Cantal" pour Chamina et "Volcan du Cantal, Pays de Saint-Flour et Ruynes-en-Margeride" pour la FFRP. Dans chacun de ces ouvrages, les Monts du Cantal sont mis à l'honneur par l'illustration et ce dès la première de couverture. Quand la FFRP choisit la vallée de la Jordanne dominée au lointain par la silhouette du Puy Griou, Chamina arrête sa décision sur l'emblématique Puy Mary, alors même que la majorité des circuits décrits dans ces deux topoguides ne se situent pas dans les Monts du Cantal. Leur surreprésentation iconographique se poursuit tout au long des ouvrages. Dans "Le Cantal", 14 des 33 photographies de paysage les représentent; on atteint un ratio de 7 sur 9 pour le topoguide de la FFRP. Les Monts du Cantal constituent bien la vitrine du département, celle que les pouvoirs publics comme les éditeurs aiment à mettre en exergue car ils correspondent à l'image de la nature qui l'on imagine plaire aux randonneurs que l'on souhaite courtiser.

Encore faudrait-il nuancer car tous les sommets ne présentent pas d'égales qualités photogéniques. Ainsi le Puy Mary et le Puy Griou, grâce à leurs lignes impeccablement pyramidales, sont très souvent mis à contribution (figure 4) alors que le Plomb du Cantal, plus arrondi et indistinct, retient moins l'intérêt des éditeurs bien qu'il s'agisse du point culminant du département $(1855 \mathrm{~m})$. C'est à une naturalisation des lieux que l'on assiste, ainsi qu'à une entreprise de séduction de la clientèle ; et celles-ci devraient s'accentuer, si l'on en croit l'évolution des éditions Chamina. Ainsi, dans une réédition simplifiée du topoguide "Le Cantal" parue en 2010, la part de l'iconographie consacrée aux paysages naturels s'est considérablement accrue au détriment des illustrations représentant le patrimoine culturel (tableau 5). 
Figure 4. Evolution des couvertures des topoguides Chamina.

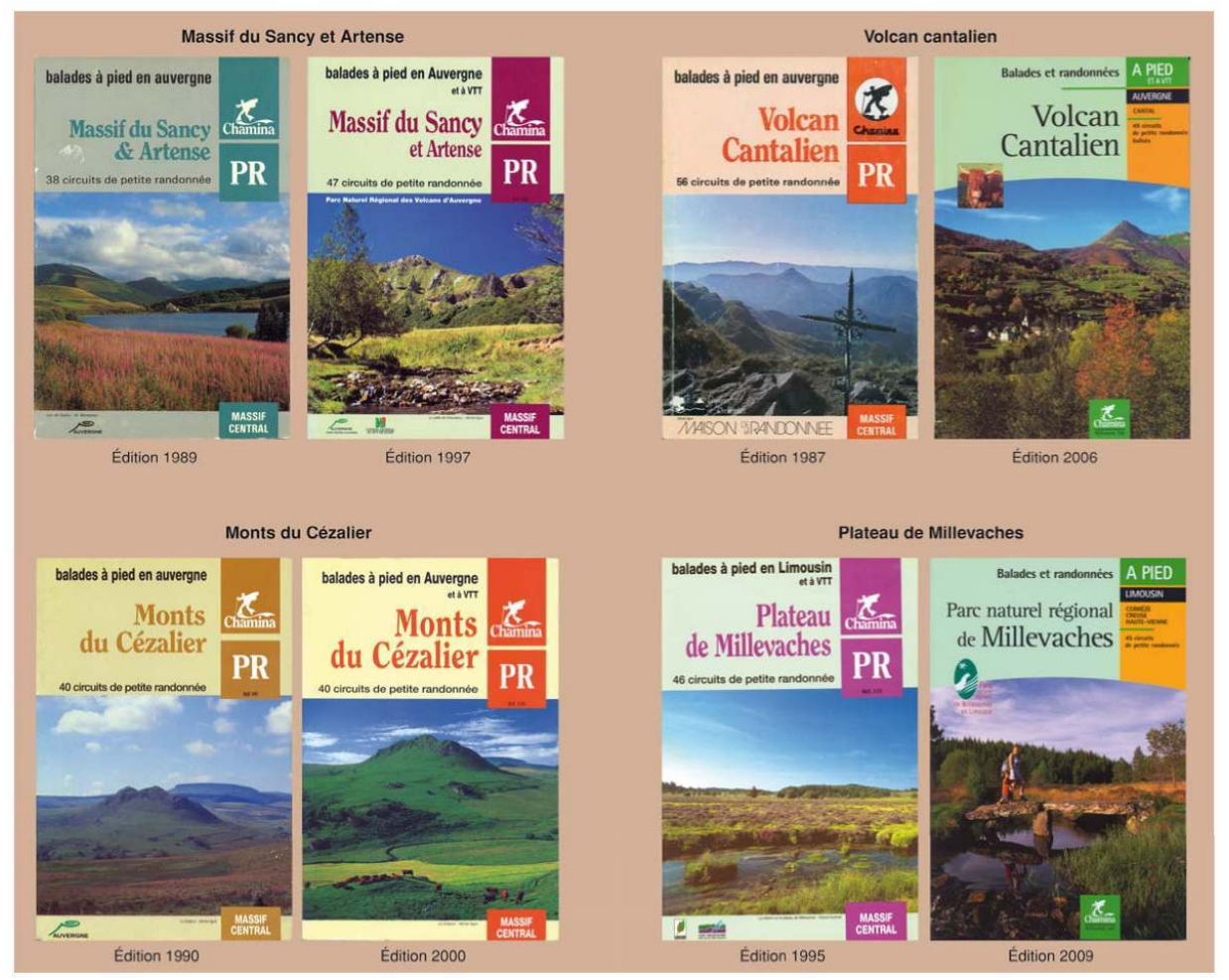

L'évolution dans le choix des photographies en pages de couverture des topoguides Chamina est également significative de la mise en œuvre progressive de principes normatifs ainsi que de la volonté d'offrir au consommateur l'image supposée lui être la plus plaisante (figure 4). Jusqu'au milieu des années 90, l'illustration retenue révèle, du Massif du Sancy à celui du Cézalier, du Volcan cantalien au plateau de Millevaches, une commune poétique de l'austérité montagnarde que n'aurait pas reniée Jean Ferrat. Photographes, secrétaires d'édition, responsables politiques, toute la chaîne de décision se montre alors sensible aux grands espaces quasiment désertiques et à la nostalgie des bruyères en fleurs.

Après 1995, tout change, le ciel est plus bleu et l'herbe plus verte. Le contraste est particulièrement frappant dans le cas du topoguide Monts du Cézalier car sont proposées pour un même site (Le Chabrut) deux représentations aux atmosphères bien distinctes. Les progrès techniques qui ont permis aux infographistes de jouer avec les couleurs qu'ils se plaisent à saturer expliquent en partie cette évolution. Mais le choix des sujets a aussi varié ; l'iconographie renonce à montrer une nature trop rude et "sauvage" ; un troupeau de vaches apparaît dans le Cézalier ; si le Puy Griou conserve la vedette en couverture du "Volcan cantalien", le village de Saint-Jacques-des-Blats émergeant d'un paisible bocage a remplacé les versants rocheux du cliché de 1987, pris depuis le Puy Mary; quant au topoguide sur Millevaches, il s'apparente désormais clairement à un dépliant touristique, avec la mise en scène d'une petite famille randonnant près de Saint-Merd-les-Oussines. Ce n'est plus seulement une certaine image de la montagne qui est donnée à voir, pour alimenter la rêverie, mais un terrain de jeux qui est proposé à la consommation de la clientèle touristique que l'on veut attirer. 


\section{Conclusion : des topoguides à double lecture} naturalisant la campagne perçue "comme lieu protégé des oppositions sociales et affranchi des exigences productives" (Chamboredon, 1985) tout en les réinscrivant par le texte dans leurs héritages culturels. D'évidence ces deux messages s'adressent à des publics différents, aux habitants des lieux et aux touristes les pratiquant de façon temporaire. Si les topoguides de l'éditeur associatif Chamina ont tout particulièrement contribué à la valorisation du patrimoine vernaculaire, et par cet intermédiaire à la construction identitaire de territoires politiques, ils ont aussi commencé à participer à la naturalisation des lieux par les images qu'ils en ont diffusées.

Certes, il s'agissait bien de développer des activités économiques, d'où la nécessité de répondre à la demande des randonneurs d'origine majoritairement urbaine. Or ceux-ci viennent chercher dans le Cantal, et plus généralement dans le Massif central, une certaine qualité des paysages, de l'environnement, une nature préservée en raison de la très faible urbanisation: "la vraie nature", "une région qui a gardé sa beauté originelle", "le côté sauvage" indiquent les personnes interrogées (Foussat, 2004). On peut toutefois se demander si ce "pas de deux" entre l'ici et l'ailleurs (Bourdeau, Mao, Corneloup, 2011) ne masque pas de grandes incompréhensions entre imaginaires endogènes et exogènes, source de potentielles conflictualités. Parfois les randonneurs, à qui les topoguides ont promis les "grands espaces", se heurtent aux barbelés des clôtures; parfois les agriculteurs doivent passer du temps à rechercher leurs bêtes égarées parce qu'une barrière a été mal refermée. Certes, les conflits d'usage ne sont pas systématiques et $P$. Vitte souligne "qu'ils relèvent bien souvent davantage d'une opposition psychologique et de modes de pensée plutôt que de réels problèmes de cohabitation" (Vitte, 2001). Toutefois les topoguides de randonnée, porteurs d'un discours ambivalent sur la manière de regarder et percevoir la moyenne montagne, contribuent à alimenter le quiproquo entre ruraux et citadins.

\section{BIBLIOGRAPHIE}

BEDARD M., AUGUSTIN J-P. et DESNOILLES R. (éd.) (2012), L'imaginaire géographique. Perspectives, pratiques et devenirs, Québec, collection Géographie Contemporaine, Presses de l'Université du Québec, 376 p.

BOURDEAU Ph., MAO P., CORNELOUP J. (2011), “Les sports de nature comme médiateurs du 'pas de deux' ville-montagne. Une habitabilité en devenir ?, Annales de Géographie, 680, pp. 449-460.

BOURDEAU Ph. (1995), “Tourisme diffus et développement territorial : le cas du tourisme sportif de nature”, in JAMOT C. et VITTE P. (éd.) (1995), Le tourisme diffus, Clermont-Ferrand, collection CERAMAC, Presses Universitaires Blaise Pascal, pp. 73-88.

Belgeo, 3 | 2012 
CHAMBOREDON J.-C. (1985), "La naturalisation de la campagne : une autre manière de cultiver les simples ?", in CADORET A. (dir.), Protection de la nature. Histoire et idéologie. De la nature à l'environnement, L'Harmattan, Paris, pp. 138-152.

CORNELOUP J. et PERRIN-MALTERRE C. (2009), "Processus de développement des loisirs sportifs de nature en Auvergne”, in LARDON S., VOLLET D., RIEUTORT L., DEVES C. et MAMDY J-F. (éd.), "Développement, attractivité et ingénierie des territoires, des enjeux de recherche pour l'action et la formation", Revue d'Auvergne, 590-591, pp. 215-232.

DERIOZ P. (2010), "Les ambiguïtés de la patrimonialisation des 'paysages naturels'”, in BOUISSET C. et DEGREMONT I. (éd.), “Patrimoines Naturels”. Sud-Ouest Européen, 30, pp. 19-36.

DUVAL M. (2007), Dynamiques spatiales et enjeux territoriaux des processus de patrimonialisation et de développement touristiques : étude comparée des gorges de l'Ardèche et du karst slovène, thèse de géographie, Université de Savoie, 516 p.

ETCHEVERRIA O. (1999), Les chemins ruraux et leur valorisation touristico-culturelle; l'exemple du Pays Basque, Université Paris I, thèse de géographie, 331 pages.

FOUSSAT M. (2004), La randonnée pédestre dans le Cantal, mémoire de maîtrise de géographie, Clermont-Ferrand, $114 \mathrm{p}$.

GAUCHON C. (2010), Tourisme et patrimoine. Un creuset pour les territoires ?, Habilitation à Diriger des Recherches, Université de Savoie, 211 p.

GRAVARI-BARBAS M., GUICHARD-ANGUIS S. (dir.) (2003), Regards croisés sur le patrimoine dans le monde à l'aube du XXI siècle. Introduction, Presses de l'Université Paris-Sorbonne, pp. 9-22.

GREVECHE M.-P. (2002), Le Plan départemental des itinéraires de promenade et randonnée : guide technique, FFRP, Paris, $152 \mathrm{p}$.

GUICHARD-ANGUIS S. et HERITIER S. (dir.) (2008), "Le Patrimoine naturel, entre culture et ressource", Géographie et cultures, 66, 149 p.

HASCHAR-NOE N. (2009), "L'aménagement des chemins de randonnée : un instrument d'identification et de 'gouvernance' territoriales”, Espaces et sociétés, 138, pp. 115-133.

HEINICH N. (2009), La fabrique du patrimoine. De la cathédrale à la petite cuillère, Ethnologie de France, Paris, 286 p.

JEUDY H.-P. (2008), La Machine patrimoniale, Belval, Circé, 123 p.

KNAFOU R. (1995), “Incertitudes, paradoxes et ambiguïtés du tourisme diffus”, in JAMOT C. et VITTE P. (éd.) (1995), Le tourisme diffus, Clermont-Ferrand, collection CERAMAC, Presses Universitaires Blaise Pascal, pp. 5-16.

LANDEL P.A. (2007), "Invention de patrimoines et construction des territoires", in GUMUCHIAN H. et PECQUEUR B. (éd.), La ressource territoriale, Paris, Economica, Anthropos, pp. 157-176.

NIEL A. et SIROST O. (2008), "Pratiques sportives et mises en paysage (Alpes, Calanques marseillaises)”, Etudes rurales, 181, pp. 181-202.

SENIL N. (2011), “Réordonner l'espace et le temps”, Revue de géographie alpine/Journal of Alpine Research [Enligne], 99, 2, mis en ligne le 20 juillet 2011, consulté le 22 août 2012, URL : http:// rga.revues.org/1436, DOI :10.4000/rga.1436

VITTE P. (2001), “Tourisme d'espaces et enjeux professionnels”, Les métiers du sport et du tourisme dans les espaces ruraux et montagnards, Montagnes Méditerranéennes, pp. 15-18.

Sources primaires 
Topoguides Chamina :

Collectif (2000), Monts du Cézalier, Chamina, Clermont-Ferrand, 111 p.

Collectif (2001), Le Cantal, Chamina, Clermont-Ferrand, 144 p.

Collectif (2001), L'Aubrac, Chamina, Clermont-Ferrand, 144 p.

Collectif (2002), Le volcan cantalien, Chamina, Clermont-Ferrand, $144 \mathrm{p}$.

Collectif (2003), Margeride et Gévaudan, Chamina, Clermont-Ferrand, 144 p.

Collectif (2010), Le Cantal, cour de massif, Chamina, Clermont-Ferrand, 54 p.

Topoguides de la FFRP :

Collectif (1995), Hauts plateaux et Monts d'Auvergne, FFRP, Paris, 80 p.

Collectif (2007), Volcan du Cantal, Pays de Saint-Flour et Ruynes-en-Margeride, FFRP, Paris, 144 p.

Topoguides de la collection départementale : "Promenade et randonnée au pays des grands espaces" du conseil général du Cantal:

Collectif (2000), Le pays entre Dordogne et Monts du Cantal, Aurillac, 100 p.

Collectif (2000), Pays de la Châtaigneraie, Aurillac, 100 p.

Collectif (2000), Le pays d'Aurillac, Aurillac, 58 p.

Collectif (2000), Entre Plomb du Cantal et Aubrac, Aurillac, 34 p.

Collectif (2000), Pays de Massiac, Aurillac, 49 p.

Collectif (2001), Le Plomb du Cantal et ses vallées, Aurillac, 92 p.

Collectif (2001), Pays de la Pinatelle et de la Haute Santoire, Aurillac, 36 p.

Collectif (2002), Pays de Saint-Flour, Aurillac, 48 p.

Collectif (2004), Les chemins des écoliers entre Margeride et Truyère, Aurillac, 51 p.

Collectif (2005), Volcans et vallées en Carladès, Aurillac, 36 p.

\section{NOTES}

1. En fait il existe aux éditions Chamina d'autres topoguides thématiques (L'eau en Auvergne, Le Volcanisme en Auvergne...) que nous n'avons pas utilisés dans ce décompte car ils reprennent des balades déjà publiées dans les topoguides ici mentionnés.

2. La liste complète est donnée dans la bibliographie.

3. La maison d'édition associative a été reprise par une entreprise qui ne crée plus beaucoup d'ouvrages originaux mais se contente de faire des compilations à partir du fond existant.

4. Avant ses difficultés financières, Chamina employait une vingtaine de permanents, ainsi qu'un important réseau d'intervenants extérieurs : auteurs, photographes, illustrateurs... Toutefois, le caractère associatif demeure: l'association "Les Amis de Chamina", présente essentiellement dans le Puy-de-Dôme, regroupe environ 200 bénévoles qui oeuvrent pour l'entretien et le balisage des sentiers de randonnée. 


\section{RÉSUMÉS}

Le présent article se propose d'analyser les représentations du Cantal, département rural de moyenne montagne situé dans le Massif central, au travers des topoguides publiés par divers éditeurs, locaux et nationaux, et notamment Chamina qui fut l'un des acteurs majeurs du développement de la randonnée, du tourisme vert et de la redécouverte du patrimoine vernaculaire dans le Massif Central. En effet, les topoguides ne se contentent pas de proposer des itinéraires, ils fournissent aussi à leurs utilisateurs une documentation parfois très riche pour appréhender les patrimoines locaux, naturels comme culturels. Ils jouent alors un rôle ambivalent, participant tout autant à naturaliser les lieux par la construction d'une image montagnarde, essentiellement destinée aux touristes, qu'à réaffirmer des identités ancrées dans la culture et l'histoire locales.

The present article suggests analyzing the representations of Cantal (which is a rural, low mountain range department, located in Massif Central), in various published topographical, in particular Chamina who was one of the major actors in the development of hiking and green tourism in the area. Chamina also focuses on the valorization of local heritage sites in the Massif Central. Thus the topographical guides do not only suggest itineraries, they also supply users with detailed documents explaining the local natural as well as cultural heritages. They thus play an ambivalent role, contributing just as much to "naturalizing" places by the construction of images of a mountain life essentially intended for tourists as to reaffirming identities anchored in the local culture and history.

\section{INDEX}

Keywords : guide, hiking, patrimonialization, mountain, representations, local development, Massif Central, Cantal

Mots-clés : guide, randonnée, patrimonialisation, montagne, représentations, développement local, Massif Central, Cantal

\section{AUTEUR}

\section{MAURICETTE FOURNIER}

maître de conférences en géographie, Université Blaise Pascal, CERAMAC, EA 997, Maison des Sciences de l'Homme, 4 rue Ledru, 63057 Clermont-Ferrand Cedex 1, Mauricette.Fournier@univbpclermont.fr 